\title{
Erratum to: Study of dijet events with a large rapidity gap between the two leading jets in pp collisions at $\sqrt{s}=7 \mathrm{TeV}$
}

\author{
CMS Collaboration* \\ CERN, 1211 Geneva 23, Switzerland
}

Received: 13 April 2020 / Accepted: 17 April 2020 / Published online: 18 May 2020

(C) CERN for the benefit of the CMS collaboration 2020

\section{Erratum to: Eur Phys J C (2018) 78:242 https://doi.org/10.1140/epjc/s10052-018-5691-6}

This erratum corrects the dedication in the PDF version of the original published manuscript: diractive processes should be diffractive processes.

\begin{abstract}
Events with no charged particles produced between the two leading jets are studied in proton-proton collisions at $\sqrt{s}=7 \mathrm{TeV}$. The jets were required to have transverse momentum $p_{\mathrm{T}}^{\text {jet }}>40 \mathrm{GeV}$ and pseudorapidity $1.5<\left|\eta^{\text {jet }}\right|<$ 4.7 , and to have values of $\eta^{\text {jet }}$ with opposite signs. The data used for this study were collected with the CMS detector during low-luminosity running at the LHC, and correspond to an integrated luminosity of $8 \mathrm{pb}^{-1}$. Events with no charged particles with $p_{\mathrm{T}}>0.2 \mathrm{GeV}$ in the interval $-1<\eta<1$ between the jets are observed in excess of calculations that assume no color-singlet exchange. The fraction of events with such a rapidity gap, amounting to $0.5-1 \%$ of the selected dijet sample, is measured as a function of the $p_{\mathrm{T}}$ of the
\end{abstract}

second-leading jet and of the rapidity separation between the jets. The data are compared to previous measurements at the Tevatron, and to perturbative quantum chromodynamics calculations based on the Balitsky-Fadin-Kuraev-Lipatov evolution equations, including different models of the nonperturbative gap survival probability.

Open Access This article is licensed under a Creative Commons Attribution 4.0 International License, which permits use, sharing, adaptation, distribution and reproduction in any medium or format, as long as you give appropriate credit to the original author(s) and the source, provide a link to the Creative Commons licence, and indicate if changes were made. The images or other third party material in this article are included in the article's Creative Commons licence, unless indicated otherwise in a credit line to the material. If material is not included in the article's Creative Commons licence and your intended use is not permitted by statutory regulation or exceeds the permitted use, you will need to obtain permission directly from the copyright holder. To view a copy of this licence, visit http://creativecomm ons.org/licenses/by/4.0/.

Funded by $\mathrm{SCOAP}^{3}$.
The original article can be found online at https://doi.org/10.1140/ epjc/s10052-018-5691-6.

We dedicate this paper to the memory of our colleague and friend Sasha Proskuryakov, who started this analysis but passed away before it was completed. His contribution to the study of diffractive processes at CMS is invaluable.

*e-mail: cms-publication-committee-chair@ cern.ch 


\section{CMS Collaboration}

Yerevan Physics Institute, Yerevan, Armenia

A. M. Sirunyan, A. Tumasyan

\section{Institut für Hochenergiephysik, Vienna, Austria}

W. Adam, E. Asilar, T. Bergauer, J. Brandstetter, E. Brondolin, M. Dragicevic, J. Erö, M. Flechl, M. Friedl, R. Frühwirth ${ }^{1}$, V. M. Ghete, C. Hartl, N. Hörmann, J. Hrubec, M. Jeitler ${ }^{1}$, A. König, I. Krätschmer, D. Liko, T. Matsushita, I. Mikulec,

D. Rabady, N. Rad, B. Rahbaran, H. Rohringer, J. Schieck ${ }^{1}$, J. Strauss, W. Waltenberger, C.-E. Wulz ${ }^{1}$

Institute for Nuclear Problems, Minsk, Belarus

O. Dvornikov, V. Makarenko, V. Mossolov, J. Suarez Gonzalez, V. Zykunov

National Centre for Particle and High Energy Physics, Minsk, Belarus

N. Shumeiko

\section{Universiteit Antwerpen, Antwerp, Belgium}

S. Alderweireldt, E. A. De Wolf, X. Janssen, J. Lauwers, M. Van De Klundert, H. Van Haevermaet, P. Van Mechelen,

N. Van Remortel, A. Van Spilbeeck

\section{Vrije Universiteit Brussel, Brussels, Belgium}

S. Abu Zeid, F. Blekman, J. D’Hondt, N. Daci, I. De Bruyn, K. Deroover, S. Lowette, S. Moortgat, L. Moreels,

A. Olbrechts, Q. Python, K. Skovpen, S. Tavernier, W. Van Doninck, P. Van Mulders, I. Van Parijs

Université Libre de Bruxelles, Brussels, Belgium

H. Brun, B. Clerbaux, G. De Lentdecker, H. Delannoy, G. Fasanella, L. Favart, R. Goldouzian, A. Grebenyuk,

G. Karapostoli, T. Lenzi, A. Léonard, J. Luetic, T. Maerschalk, A. Marinov, A. Randle-conde, T. Seva, C. Vander Velde,

P. Vanlaer, D. Vannerom, R. Yonamine, F. Zenoni, F. Zhang ${ }^{2}$

\section{Ghent University, Ghent, Belgium}

A. Cimmino, T. Cornelis, D. Dobur, A. Fagot, M. Gul, I. Khvastunov, D. Poyraz, S. Salva, R. Schöfbeck, M. Tytgat, W. Van Driessche, E. Yazgan, N. Zaganidis

\section{Université Catholique de Louvain, Louvain-la-Neuve, Belgium}

H. Bakhshiansohi, C. Beluffi ${ }^{3}$, O. Bondu, S. Brochet, G. Bruno, A. Caudron, S. De Visscher, C. Delaere, M. Delcourt,

B. Francois, A. Giammanco, A. Jafari, M. Komm, G. Krintiras, V. Lemaitre, A. Magitteri, A. Mertens, M. Musich,

K. Piotrzkowski, L. Quertenmont, M. Selvaggi, M. Vidal Marono, S. Wertz

\section{Université de Mons, Mons, Belgium}

N. Beliy

Centro Brasileiro de Pesquisas Fisicas, Rio de Janeiro, Brazil

W. L. Aldá Júnior, F. L. Alves, G. A. Alves, L. Brito, C. Hensel, A. Moraes, M. E. Pol, P. Rebello Teles

Universidade do Estado do Rio de Janeiro, Rio de Janeiro, Brazil

E. Belchior Batista Das Chagas, W. Carvalho, J. Chinellato ${ }^{4}$, A. Custódio, E. M. Da Costa, G. G. Da Silveira ${ }^{5}$,

D. De Jesus Damiao, C. De Oliveira Martins, S. Fonseca De Souza, L. M. Huertas Guativa, H. Malbouisson,

D. Matos Figueiredo, C. Mora Herrera, L. Mundim, H. Nogima, W. L. Prado Da Silva, A. Santoro, A. Sznajder,

E. J. Tonelli Manganote ${ }^{4}$, F. Torres Da Silva De Araujo, A. Vilela Pereira

Universidade Estadual Paulista ${ }^{a}$, Universidade Federal do $\mathrm{ABC}^{b}$, São Paulo, Brazil

S. Ahuja ${ }^{a}$, C. A. Bernardes ${ }^{a}$, S. Dogra ${ }^{a}$, T. R. Fernandez Perez Tomei ${ }^{a}$, E. M. Gregores ${ }^{b}$, P. G. Mercadante ${ }^{b}$, C. S. Moon ${ }^{a}$, S. F. Novaes ${ }^{a}$, Sandra S. Padula ${ }^{a}$, D. Romero $\operatorname{Abad}^{b}$, J. C. Ruiz Vargas ${ }^{a}$

Institute for Nuclear Research and Nuclear Energy of Bulgaria Academy of Sciences, Sofia, Bulgaria

A. Aleksandrov, R. Hadjiiska, P. Iaydjiev, M. Rodozov, S. Stoykova, G. Sultanov, M. Vutova

University of Sofia, Sofia, Bulgaria

A. Dimitrov, I. Glushkov, L. Litov, B. Pavlov, P. Petkov 
Beihang University, Beijing, China

W. Fang ${ }^{6}$

Institute of High Energy Physics, Beijing, China

M. Ahmad, J. G. Bian, G. M. Chen, H. S. Chen, M. Chen, Y. Chen ${ }^{7}$, T. Cheng, C. H. Jiang, D. Leggat, Z. Liu, F. Romeo, M. Ruan, S. M. Shaheen, A. Spiezia, J. Tao, C. Wang, Z. Wang, H. Zhang, J. Zhao

State Key Laboratory of Nuclear Physics and Technology, Peking University, Beijing, China

Y. Ban, G. Chen, Q. Li, S. Liu, Y. Mao, S. J. Qian, D. Wang, Z. Xu

Universidad de Los Andes, Bogotá, Colombia

C. Avila, A. Cabrera, L. F. Chaparro Sierra, C. Florez, J. P. Gomez, C. F. González Hernández, J. D. Ruiz Alvarez ${ }^{8}$,

J. C. Sanabria

Faculty of Electrical Engineering, Mechanical Engineering and Naval Architecture, University of Split, Split, Croatia N. Godinovic, D. Lelas, I. Puljak, P. M. Ribeiro Cipriano, T. Sculac

University of Split, Faculty of Science, Split, Croatia

Z. Antunovic, M. Kovac

Institute Rudjer Boskovic, Zagreb, Croatia

V. Brigljevic, D. Ferencek, K. Kadija, B. Mesic, T. Susa

University of Cyprus, Nicosia, Cyprus

M. W. Ather, A. Attikis, G. Mavromanolakis, J. Mousa, C. Nicolaou, F. Ptochos, P. A. Razis, H. Rykaczewski

Charles University, Prague, Czech Republic

M. Finger ${ }^{9}$, M. Finger Jr. ${ }^{9}$

Universidad San Francisco de Quito, Quito, Ecuador

E. Carrera Jarrin

Academy of Scientific Research and Technology of the Arab Republic of Egypt, Egyptian Network of High Energy Physics, Cairo, Egypt

S. Elgammal ${ }^{10}$, A. Ellithi Kamel ${ }^{11}$, A. Mohamed ${ }^{12}$

National Institute of Chemical Physics and Biophysics, Tallinn, Estonia

M. Kadastik, L. Perrini, M. Raidal, A. Tiko, C. Veelken

Department of Physics, University of Helsinki, Helsinki, Finland

P. Eerola, J. Pekkanen, M. Voutilainen

Helsinki Institute of Physics, Helsinki, Finland

J. Härkönen, T. Järvinen, V. Karimäki, R. Kinnunen, T. Lampén, K. Lassila-Perini, S. Lehti, T. Lindén, P. Luukka,

J. Tuominiemi, E. Tuovinen, L. Wendland

Lappeenranta University of Technology, Lappeenranta, Finland

J. Talvitie, T. Tuuva

IRFU, CEA, Université Paris-Saclay, Gif-sur-Yvette, France

M. Besancon, F. Couderc, M. Dejardin, D. Denegri, B. Fabbro, J. L. Faure, C. Favaro, F. Ferri, S. Ganjour, S. Ghosh, A. Givernaud, P. Gras, G. Hamel de Monchenault, P. Jarry, I. Kucher, E. Locci, M. Machet, J. Malcles, J. Rander,

A. Rosowsky, M. Titov

Laboratoire Leprince-Ringuet, Ecole polytechnique, CNRS/IN2P3, Université Paris-Saclay, Palaiseau, France A. Abdulsalam, I. Antropov, S. Baffioni, F. Beaudette, P. Busson, L. Cadamuro, E. Chapon, C. Charlot, O. Davignon, R. Granier de Cassagnac, M. Jo, S. Lisniak, P. Miné, M. Nguyen, C. Ochando, G. Ortona, P. Paganini, P. Pigard,

S. Regnard, R. Salerno, Y. Sirois, A. G. Stahl Leiton, T. Strebler, Y. Yilmaz, A. Zabi, A. Zghiche 
Université de Strasbourg, CNRS IPHC UMR 7178, 67000 Strasbourg, France

J.-L. Agram ${ }^{13}$, J. Andrea, D. Bloch, J.-M. Brom, M. Buttignol, E. C. Chabert, N. Chanon, C. Collard, E. Conte ${ }^{13}$, X. Coubez, J.-C. Fontaine ${ }^{13}$, D. Gelé, U. Goerlach, A.-C. Le Bihan, P. Van Hove

Centre de Calcul de l'Institut National de Physique Nucleaire et de Physique des Particules, CNRS/IN2P3, Villeurbanne, France

S. Gadrat

Université de Lyon, Université Claude Bernard Lyon 1, CNRS-IN2P3, Institut de Physique Nucléaire de Lyon, Villeurbanne, France

S. Beauceron, C. Bernet, G. Boudoul, C. A. Carrillo Montoya, R. Chierici, D. Contardo, B. Courbon, P. Depasse, H. El Mamouni, J. Fay, S. Gascon, M. Gouzevitch, G. Grenier, B. Ille, F. Lagarde, I. B. Laktineh, M. Lethuillier, L. Mirabito, A. L. Pequegnot, S. Perries, A. Popov ${ }^{14}$, V. Sordini, M. Vander Donckt, P. Verdier, S. Viret

\section{Georgian Technical University, Tbilisi, Georgia}

T. Toriashvili ${ }^{15}$

Tbilisi State University, Tbilisi, Georgia

Z. Tsamalaidze ${ }^{9}$

RWTH Aachen University, I. Physikalisches Institut, Aachen, Germany

C. Autermann, S. Beranek, L. Feld, M. K. Kiesel, K. Klein, M. Lipinski, M. Preuten, C. Schomakers, J. Schulz, T. Verlage

RWTH Aachen University, III. Physikalisches Institut A, Aachen, Germany

A. Albert, M. Brodski, E. Dietz-Laursonn, D. Duchardt, M. Endres, M. Erdmann, S. Erdweg, T. Esch, R. Fischer, A. Güth, M. Hamer, T. Hebbeker, C. Heidemann, K. Hoepfner, S. Knutzen, M. Merschmeyer, A. Meyer, P. Millet, S. Mukherjee, M. Olschewski, K. Padeken, T. Pook, M. Radziej, H. Reithler, M. Rieger, F. Scheuch, L. Sonnenschein, D. Teyssier, S. Thüer

\section{RWTH Aachen University, III. Physikalisches Institut B, Aachen, Germany}

V. Cherepanov, G. Flügge, B. Kargoll, T. Kress, A. Künsken, J. Lingemann, T. Müller, A. Nehrkorn, A. Nowack, C. Pistone, O. Pooth, A. Stahl ${ }^{16}$

\section{Deutsches Elektronen-Synchrotron, Hamburg, Germany}

M. Aldaya Martin, T. Arndt, C. Asawatangtrakuldee, K. Beernaert, O. Behnke, U. Behrens, A. A. Bin Anuar, K. Borras ${ }^{17}$, A. Campbell, P. Connor, C. Contreras-Campana, F. Costanza, C. Diez Pardos, G. Dolinska, G. Eckerlin, D. Eckstein, T. Eichhorn, E. Eren, E. Gallo ${ }^{18}$, J. Garay Garcia, A. Geiser, A. Gizhko, J. M. Grados Luyando, A. Grohsjean,

P. Gunnellini, A. Harb, J. Hauk, M. Hempel ${ }^{19}$, H. Jung, A. Kalogeropoulos, O. Karacheban ${ }^{19}$, M. Kasemann, J. Keaveney,

C. Kleinwort, I. Korol, D. Krücker, W. Lange, A. Lelek, T. Lenz, J. Leonard, K. Lipka, A. Lobanov, W. Lohmann ${ }^{19}$,

R. Mankel, I.-A. Melzer-Pellmann, A. B. Meyer, G. Mittag, J. Mnich, A. Mussgiller, D. Pitzl, R. Placakyte, A. Raspereza,

B. Roland, M. Ö. Sahin, P. Saxena, T. Schoerner-Sadenius, S. Spannagel, N. Stefaniuk, G. P. Van Onsem, R. Walsh,

C. Wissing

\section{University of Hamburg, Hamburg, Germany}

V. Blobel, M. Centis Vignali, A. R. Draeger, T. Dreyer, E. Garutti, D. Gonzalez, J. Haller, M. Hoffmann, A. Junkes,

R. Klanner, R. Kogler, N. Kovalchuk, T. Lapsien, I. Marchesini, D. Marconi, M. Meyer, M. Niedziela, D. Nowatschin,

F. Pantaleo ${ }^{16}$, T. Peiffer, A. Perieanu, C. Scharf, P. Schleper, A. Schmidt, S. Schumann, J. Schwandt, H. Stadie,

G. Steinbrück, F. M. Stober, M. Stöver, H. Tholen, D. Troendle, E. Usai, L. Vanelderen, A. Vanhoefer, B. Vormwald

\section{Institut für Experimentelle Kernphysik, Karlsruhe, Germany}

M. Akbiyik, C. Barth, S. Baur, C. Baus, J. Berger, E. Butz, R. Caspart, T. Chwalek, F. Colombo, W. De Boer,

A. Dierlamm, S. Fink, B. Freund, R. Friese, M. Giffels, A. Gilbert, P. Goldenzweig, D. Haitz, F. Hartmann ${ }^{16}$, S. M. Heindl,

U. Husemann, F. Kassel ${ }^{16}$, I. Katkov ${ }^{14}$, S. Kudella, H. Mildner, M. U. Mozer, Th. Müller, M. Plagge, G. Quast,

K. Rabbertz, S. Röcker, F. Roscher, M. Schröder, I. Shvetsov, G. Sieber, H. J. Simonis, R. Ulrich, S. Wayand, M. Weber,

T. Weiler, S. Williamson, C. Wöhrmann, R. Wolf

Institute of Nuclear and Particle Physics (INPP), NCSR Demokritos, Aghia Paraskevi, Greece

G. Anagnostou, G. Daskalakis, T. Geralis, V. A. Giakoumopoulou, A. Kyriakis, D. Loukas, I. Topsis-Giotis 
National and Kapodistrian University of Athens, Athens, Greece

S. Kesisoglou, A. Panagiotou, N. Saoulidou, E. Tziaferi

University of Ioánnina, Ioánnina, Greece

I. Evangelou, G. Flouris, C. Foudas, P. Kokkas, N. Loukas, N. Manthos, I. Papadopoulos, E. Paradas

MTA-ELTE Lendület CMS Particle and Nuclear Physics Group, Eötvös Loránd University, Budapest, Hungary

N. Filipovic, G. Pasztor

Wigner Research Centre for Physics, Budapest, Hungary

G. Bencze, C. Hajdu, D. Horvath ${ }^{20}$, F. Sikler, V. Veszpremi, G. Vesztergombi ${ }^{21}$, A. J. Zsigmond

Institute of Nuclear Research ATOMKI, Debrecen, Hungary

N. Beni, S. Czellar, J. Karancsi ${ }^{22}$, A. Makovec, J. Molnar, Z. Szillasi

Institute of Physics, University of Debrecen, Debrecen, Hungary

M. Bartók ${ }^{21}$, P. Raics, Z. L. Trocsanyi, B. Ujvari

Indian Institute of Science (IISc), Bangalore, India

J. R. Komaragiri

National Institute of Science Education and Research, Bhubaneswar, India

S. Bahinipati ${ }^{23}$, S. Bhowmik ${ }^{24}$, S. Choudhury ${ }^{25}$, P. Mal, K. Mandal, A. Nayak ${ }^{26}$, D. K. Sahoo ${ }^{23}$, N. Sahoo, S. K. Swain

Panjab University, Chandigarh, India

S. Bansal, S. B. Beri, V. Bhatnagar, U. Bhawandeep, R. Chawla, A. K. Kalsi, A. Kaur, M. Kaur, R. Kumar, P. Kumari,

A. Mehta, M. Mittal, J. B. Singh, G. Walia

University of Delhi, Delhi, India

Ashok Kumar, A. Bhardwaj, B. C. Choudhary, R. B. Garg, S. Keshri, S. Malhotra, M. Naimuddin, K. Ranjan, R. Sharma, V. Sharma

Saha Institute of Nuclear Physics, HBNI, Kolkata, India

R. Bhattacharya, S. Bhattacharya, K. Chatterjee, S. Dey, S. Dutt, S. Dutta, S. Ghosh, N. Majumdar, A. Modak, K. Mondal, S. Mukhopadhyay, S. Nandan, A. Purohit, A. Roy, D. Roy, S. Roy Chowdhury, S. Sarkar, M. Sharan, S. Thakur

Indian Institute of Technology Madras, Madras, India

P. K. Behera

Bhabha Atomic Research Centre, Mumbai, India

R. Chudasama, D. Dutta, V. Jha, V. Kumar, A. K. Mohanty ${ }^{16}$, P. K. Netrakanti, L. M. Pant, P. Shukla, A. Topkar

Tata Institute of Fundamental Research-A, Mumbai, India

T. Aziz, S. Dugad, G. Kole, B. Mahakud, S. Mitra, G. B. Mohanty, B. Parida, N. Sur, B. Sutar

Tata Institute of Fundamental Research-B, Mumbai, India

S. Banerjee, R. K. Dewanjee, S. Ganguly, M. Guchait, Sa. Jain, S. Kumar, M. Maity ${ }^{24}$, G. Majumder, K. Mazumdar, T. Sarkar ${ }^{24}$, N. Wickramage ${ }^{27}$

Indian Institute of Science Education and Research (IISER), Pune, India

S. Chauhan, S. Dube, V. Hegde, A. Kapoor, K. Kothekar, S. Pandey, A. Rane, S. Sharma

Institute for Research in Fundamental Sciences (IPM), Tehran, Iran

S. Chenarani ${ }^{28}$, E. Eskandari Tadavani, S. M. Etesami ${ }^{28}$, M. Khakzad, M. Mohammadi Najafabadi, M. Naseri,

S. Paktinat Mehdiabadi ${ }^{29}$, F. Rezaei Hosseinabadi, B. Safarzadeh ${ }^{30}$, M. Zeinali

University College Dublin, Dublin, Ireland

M. Felcini, M. Grunewald 
INFN Sezione di Bari ${ }^{a}$, Università di Bari ${ }^{b}$, Politecnico di Bari ${ }^{c}$, Bari, Italy

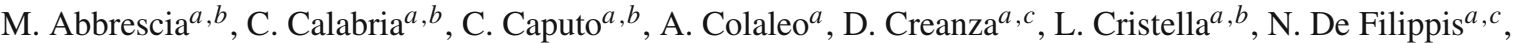

M. De Palma ${ }^{a, b}$, L. Fiore ${ }^{a}$, G. Iasellii ${ }^{a, c}$, G. Maggi ${ }^{a, c}$, M. Maggi ${ }^{a}$, G. Miniello ${ }^{a, b}$, S. My ${ }^{a, b}$, S. Nuzzo $^{a, b}$, A. Pompilia ${ }^{a, b}$,

G. Pugliese ${ }^{a, c}$, R. Radogna ${ }^{a, b}$, A. Ranieri ${ }^{a}$, G. Selvaggi ${ }^{a, b}$, A. Sharma ${ }^{a}$, L. Silvestris ${ }^{a, 16}$, R. Venditti $^{a, b}$, P. Verwilligen $^{a}$

INFN Sezione di Bologna ${ }^{a}$, Università di Bologna ${ }^{b}$, Bologna, Italy

G. Abbiendi ${ }^{a}$, C. Battilana, D. Bonacorsi ${ }^{a, b}$, S. Braibant-Giacomelli ${ }^{a, b}$, L. Brigliadori ${ }^{a, b}$, R. Campanini ${ }^{a, b}$,

P. Capiluppi ${ }^{a, b}$, A. Castro ${ }^{a, b}$, F. R. Cavallo ${ }^{a}$, S. S. Chhibra ${ }^{a, b}$, G. Codispoti $^{a, b}$, M. Cuffiani ${ }^{a, b}$, G. M. Dallavalle ${ }^{a}$,

F. Fabbri ${ }^{a}$, A. Fanfani ${ }^{a}, b$, D. Fasanella ${ }^{a}, b$, P. Giacomelli ${ }^{a}$, C. Grandi ${ }^{a}$, L. Guiducci ${ }^{a}, b$, S. Marcellini ${ }^{a}$, G. Masetti ${ }^{a}$,

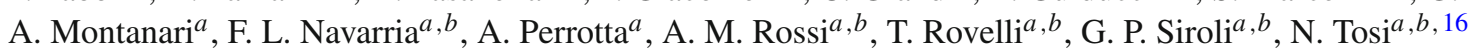

INFN Sezione di Catania ${ }^{a}$, Università di Catania ${ }^{b}$, Catania, Italy

S. Albergo ${ }^{a, b}$, S. Costa ${ }^{a, b}$, A. Di Mattia ${ }^{a}$, F. Giordano $^{a, b}$, R. Potenza $^{a, b}$, A. Tricomi $^{a, b}$, C. Tuve $^{a, b}$

INFN Sezione di Firenze ${ }^{a}$, Università di Firenze ${ }^{b}$, Firenze, Italy

G. Barbagli ${ }^{a}$, V. Ciulli ${ }^{a}, b$, C. Civinini ${ }^{a}$, R. D’Alessandro ${ }^{a, b}$, E. Focardi $^{a}, b$, P. Lenzi $^{a}, b$, M. Meschini $^{a}$, S. Paoletti $^{a}$,

L. Russo ${ }^{a, 31}$, G. Sguazzoni ${ }^{a}$, D. Strom ${ }^{a}$, L. Viliani ${ }^{a}, b, 16$

INFN Laboratori Nazionali di Frascati, Frascati, Italy

L. Benussi, S. Bianco, F. Fabbri, D. Piccolo, F. Primavera ${ }^{16}$

INFN Sezione di Genova ${ }^{a}$, Università di Genova ${ }^{b}$, Genoa, Italy

V. Calvelli ${ }^{a, b}$, F. Ferro $^{a}$, M. R. Monge ${ }^{a, b}$, E. Robutti $^{a}$, S. Tosi $^{a, b}$

INFN Sezione di Milano-Bicocca ${ }^{a}$, Università di Milano-Bicocca ${ }^{b}$, Milan, Italy

L. Brianza ${ }^{a, b}, 16$, F. Brivio ${ }^{a, b}$, V. Ciriolo, M. E. Dinardo ${ }^{a, b}$, S. Fiorendi ${ }^{a, b}, 16$, S. Gennai ${ }^{a}$, A. Ghezzi ${ }^{a, b}$, P. Govoni ${ }^{a, b}$, M. Malberti ${ }^{a}, b$, S. Malvezzi ${ }^{a}$, R. A. Manzoni ${ }^{a, b}$, D. Menasce ${ }^{a}$, L. Moroni ${ }^{a}$, M. Paganoni ${ }^{a}, b$, D. Pedrini ${ }^{a}$, S. Pigazzini $^{a}, b$, S. Ragazzi ${ }^{a, b}$, T. Tabarelli de Fatis ${ }^{a, b}$

INFN Sezione di Napoli ${ }^{a}$, Università di Napoli 'Federico II' $^{b}$, Napoli, Italy, Università della Basilicata ${ }^{c}$, Potenza, $^{\prime}$ Italy, Università G. Marconi ${ }^{d}$, Rome, Italy

S. Buontempo ${ }^{a}$, N. Cavallo ${ }^{a, c}$, G. De Nardo, S. Di Guida ${ }^{a, d}, 16$, F. Fabozzi ${ }^{a, c}$, F. Fienga ${ }^{a, b}$, A. O. M. Iorio ${ }^{a, b}$, L. Lista ${ }^{a}$, S. Meola ${ }^{a, d}, 16$, P. Paolucci ${ }^{a, 16}$, C. Sciacca ${ }^{a, b}$, F. Thyssen ${ }^{a}$

INFN Sezione di Padova ${ }^{a}$, Università di Padova ${ }^{b}$, Padova, Italy, Università di Trento $^{c}$, Trento, Italy

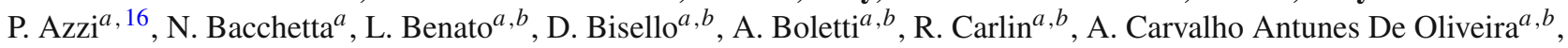

P. Checchia $^{a}$, M. Dall'Osso ${ }^{a, b}$, P. De Castro Manzano ${ }^{a}$, T. Dorigo ${ }^{a}$, U. Dosselli ${ }^{a}$, F. Gasparini $^{a}, b$, U. Gasparini ${ }^{a, b}$,

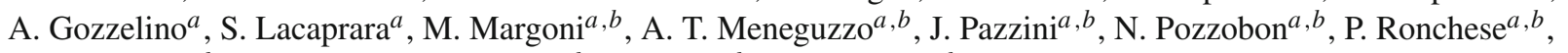

F. Simonetto ${ }^{a, b}$, E. Torassa $^{a}$, M. Zanetti ${ }^{a, b}$, P. Zotto ${ }^{a, b}$, G. Zumerle $^{a, b}$

INFN Sezione di Pavia ${ }^{a}$, Università di Pavia ${ }^{b}$, Pavia, Italy

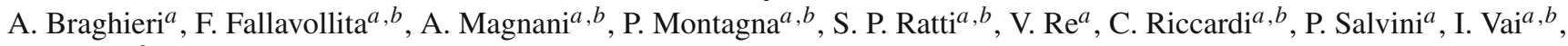
P. Vitulo ${ }^{a, b}$

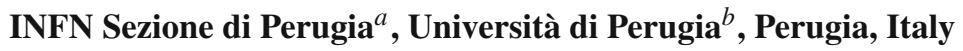

L. Alunni Solestizi ${ }^{a, b}$, G. M. Bilei ${ }^{a}$, D. Ciangottini ${ }^{a, b}$, L. Fanò $^{a, b}$, P. Lariccia $^{a, b}$, R. Leonardi $^{a, b}$, G. Mantovani $^{a, b}$, V. Mariani ${ }^{a}, b$, M. Menichelli ${ }^{a}$, A. Saha ${ }^{a}$, A. Santocchia ${ }^{a, b}$

INFN Sezione di Pisa ${ }^{a}$, Università di Pisa ${ }^{b}$, Scuola Normale Superiore di Pisa ${ }^{c}$, Pisa, Italy

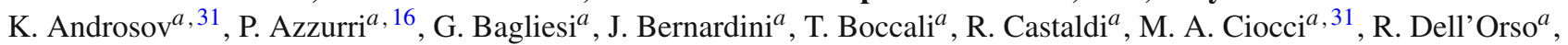

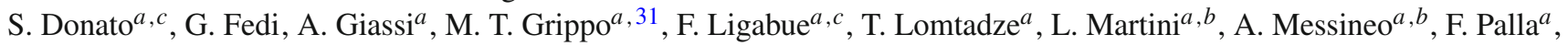
A. Rizzi ${ }^{a}, b$, A. Savoy-Navarro ${ }^{a}, 32$, P. Spagnolo $^{a}$, R. Tenchini ${ }^{a}$, G. Tonelli $^{a}, b$, A. Venturi $^{a}$, P. G. Verdini $^{a}$

INFN Sezione di Roma ${ }^{a}$, Sapienza Università di Roma ${ }^{b}$, Rome, Italy

L. Barone ${ }^{a, b}$, F. Cavallari ${ }^{a}$, M. Cipriani ${ }^{a, b}$, D. Del Re ${ }^{a, b, 16}$, M. Diemoz ${ }^{a}$, S. Gelli ${ }^{a, b}$, E. Longo ${ }^{a, b}$, F. Margaroli $^{a, b}$,

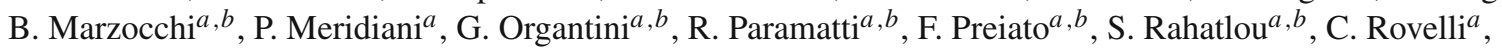

F. Santanastasio ${ }^{a, b}$ 
INFN Sezione di Torino ${ }^{a}$, Università di Torino ${ }^{b}$, Turin, Italy, Università del Piemonte Orientale ${ }^{c}$, Novara, Italy

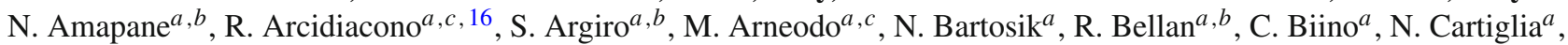
F. Cenna ${ }^{a, b}$, M. Costa ${ }^{a, b}$, R. Covarelli ${ }^{a, b}$, A. Degano ${ }^{a, b}$, N. Demaria ${ }^{a}$, L. Finco ${ }^{a, b}$, B. Kiani ${ }^{a, b}$, C. Mariotti ${ }^{a}$, S. Maselli $^{a}$,

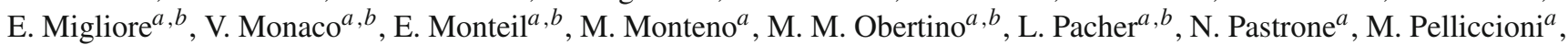
G. L. Pinna Angioni ${ }^{a, b}$, F. Ravera ${ }^{a, b}$, A. Romero ${ }^{a, b}$, M. Ruspa ${ }^{a, c}$, R. Sacchi ${ }^{a, b}$, K. Shchelina ${ }^{a, b}$, V. Sola ${ }^{a}$, A. Solano ${ }^{a, b}$, A. Staiano ${ }^{a}$, P. Traczyk ${ }^{a, b}$

INFN Sezione di Trieste ${ }^{a}$, Università di Trieste ${ }^{b}$, Trieste, Italy

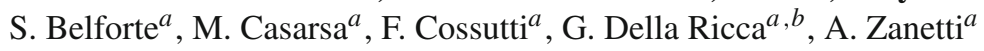

Kyungpook National University, Taegu, Korea

D. H. Kim, G. N. Kim, M. S. Kim, S. Lee, S. W. Lee, Y. D. Oh, S. Sekmen, D. C. Son, Y. C. Yang

Chonbuk National University, Chonju, Korea

A. Lee

Chonnam National University, Institute for Universe and Elementary Particles, Kwangju, Korea

H. Kim

Hanyang University, Seoul, Korea

J. A. Brochero Cifuentes, T. J. Kim

Korea University, Seoul, Korea

S. Cho, S. Choi, Y. Go, D. Gyun, S. Ha, B. Hong, Y. Jo, Y. Kim, K. Lee, K. S. Lee, S. Lee, J. Lim, S. K. Park, Y. Roh

Seoul National University, Seoul, Korea

J. Almond, J. Kim, H. Lee, S. B. Oh, B. C. Radburn-Smith, S. h. Seo, U. K. Yang, H. D. Yoo, G. B. Yu

University of Seoul, Seoul, Korea

M. Choi, H. Kim, J. H. Kim, J. S. H. Lee, I. C. Park, G. Ryu, M. S. Ryu

Sungkyunkwan University, Suwon, Korea

Y. Choi, J. Goh, C. Hwang, J. Lee, I. Yu

Vilnius University, Vilnius, Lithuania

V. Dudenas, A. Juodagalvis, J. Vaitkus

National Centre for Particle Physics, Universiti Malaya, Kuala Lumpur, Malaysia

I. Ahmed, Z. A. Ibrahim, M. A. B. Md Ali ${ }^{33}$, F. Mohamad Idris ${ }^{34}$, W. A. T. Wan Abdullah, M. N. Yusli, Z. Zolkapli

Centro de Investigacion y de Estudios Avanzados del IPN, Mexico City, Mexico

H. Castilla-Valdez, E. De La Cruz-Burelo, I. Heredia-De La Cruz ${ }^{35}$, A. Hernandez-Almada, R. Lopez-Fernandez,

R. Magaña Villalba, J. Mejia Guisao, A. Sanchez-Hernandez

Universidad Iberoamericana, Mexico City, Mexico

S. Carrillo Moreno, C. Oropeza Barrera, F. Vazquez Valencia

Benemerita Universidad Autonoma de Puebla, Puebla, Mexico

S. Carpinteyro, I. Pedraza, H. A. Salazar Ibarguen, C. Uribe Estrada

Universidad Autónoma de San Luis Potosí, San Luis Potosí, Mexico

A. Morelos Pineda

University of Auckland, Auckland, New Zealand

D. Krofcheck

University of Canterbury, Christchurch, New Zealand

P. H. Butler

National Centre for Physics, Quaid-I-Azam University, Islamabad, Pakistan

A. Ahmad, M. Ahmad, Q. Hassan, H. R. Hoorani, W. A. Khan, A. Saddique, M. A. Shah, M. Shoaib, M. Waqas 
National Centre for Nuclear Research, Swierk, Poland

H. Bialkowska, M. Bluj, B. Boimska, T. Frueboes, M. Górski, M. Kazana, K. Nawrocki, K. Romanowska-Rybinska, M. Szleper, P. Zalewski

Institute of Experimental Physics, Faculty of Physics, University of Warsaw, Warsaw, Poland

K. Bunkowski, A. Byszuk ${ }^{36}$, K. Doroba, A. Kalinowski, M. Konecki, J. Krolikowski, M. Misiura, M. Olszewski,

M. Walczak

Laboratório de Instrumentação e Física Experimental de Partículas, Lisbon, Portugal

P. Bargassa, C. Beirão Da Cruz E. Silva, B. Calpas, A. Di Francesco, P. Faccioli, M. Gallinaro, J. Hollar, N. Leonardo,

L. Lloret Iglesias, M. V. Nemallapudi, J. Seixas, O. Toldaiev, D. Vadruccio, J. Varela

Joint Institute for Nuclear Research, Dubna, Russia

S. Afanasiev, P. Bunin, M. Gavrilenko, I. Golutvin, I. Gorbunov, A. Kamenev, V. Karjavin, A. Lanev, A. Malakhov,

V. Matveev ${ }^{37,38}$, V. Palichik, V. Perelygin, S. Shmatov, S. Shulha, N. Skatchkov, V. Smirnov, N. Voytishin, A. Zarubin

Petersburg Nuclear Physics Institute, Gatchina, St. Petersburg, Russia

L. Chtchipounov, V. Golovtsov, Y. Ivanov, V. Kim ${ }^{39}$, E. Kuznetsova ${ }^{40}$, V. Murzin, V. Oreshkin, V. Sulimov, A. Vorobyev

Institute for Nuclear Research, Moscow, Russia

Yu. Andreev, A. Dermenev, S. Gninenko, N. Golubev, A. Karneyeu, M. Kirsanov, N. Krasnikov, A. Pashenkov, D. Tlisov,

A. Toropin

Institute for Theoretical and Experimental Physics, Moscow, Russia

V. Epshteyn, V. Gavrilov, N. Lychkovskaya, V. Popov, I. Pozdnyakov, G. Safronov, A. Spiridonov, M. Toms, E. Vlasov, A. Zhokin

Moscow Institute of Physics and Technology, Moscow, Russia

T. Aushev, A. Bylinkin ${ }^{38}$

National Research Nuclear University 'Moscow Engineering Physics Institute' (MEPhI), Moscow, Russia

M. Danilov ${ }^{41}$, S. Polikarpov, E. Tarkovskii

P.N. Lebedev Physical Institute, Moscow, Russia

V. Andreev, M. Azarkin ${ }^{38}$, I. Dremin ${ }^{38}$, M. Kirakosyan, A. Leonidov ${ }^{38}$, A. Terkulov

Skobeltsyn Institute of Nuclear Physics, Lomonosov Moscow State University, Moscow, Russia

A. Baskakov, A. Belyaev, E. Boos, A. Ershov, A. Gribushin, L. Khein, V. Klyukhin, O. Kodolova, I. Lokhtin, O. Lukina, I. Miagkov, S. Obraztsov, S. Petrushanko, V. Savrin, A. Snigirev

Novosibirsk State University (NSU), Novosibirsk, Russia

V. Blinov ${ }^{42}$, Y. Skovpen ${ }^{42}$, D. Shtol ${ }^{42}$

State Research Center of Russian Federation, Institute for High Energy Physics, Protvinosant, Russia

I. Azhgirey, I. Bayshev, S. Bitioukov, D. Elumakhov, V. Kachanov, A. Kalinin, D. Konstantinov, V. Krychkine, V. Petrov, R. Ryutin, A. Sobol, S. Troshin, N. Tyurin, A. Uzunian, A. Volkov

University of Belgrade, Faculty of Physics and Vinca Institute of Nuclear Sciences, Belgrade, Serbia P. Adzic ${ }^{43}$, P. Cirkovic, D. Devetak, M. Dordevic, J. Milosevic, V. Rekovic

Centro de Investigaciones Energéticas Medioambientales y Tecnológicas (CIEMAT), Madrid, Spain J. Alcaraz Maestre, M. Barrio Luna, E. Calvo, M. Cerrada, M. Chamizo Llatas, N. Colino, B. De La Cruz, A. Delgado Peris, A. Escalante Del Valle, C. Fernandez Bedoya, J. P. Fernández Ramos, J. Flix, M. C. Fouz, P. Garcia-Abia, O. Gonzalez Lopez, S. Goy Lopez, J. M. Hernandez, M. I. Josa, E. Navarro De Martino,

A. Pérez-Calero Yzquierdo, J. Puerta Pelayo, A. Quintario Olmeda, I. Redondo, L. Romero, M. S. Soares

Universidad Autónoma de Madrid, Madrid, Spain

C. Albajar, J. F. de Trocóniz, M. Missiroli, D. Moran 


\section{Universidad de Oviedo, Oviedo, Spain}

J. Cuevas, J. Fernandez Menendez, I. Gonzalez Caballero, J. R. González Fernández, E. Palencia Cortezon,

S. Sanchez Cruz, I. Suárez Andrés, P. Vischia, J. M. Vizan Garcia

\section{Instituto de Física de Cantabria (IFCA), CSIC-Universidad de Cantabria, Santander, Spain}

I. J. Cabrillo, A. Calderon, E. Curras, M. Fernandez, J. Garcia-Ferrero, G. Gomez, A. Lopez Virto, J. Marco,

C. Martinez Rivero, F. Matorras, J. Piedra Gomez, T. Rodrigo, A. Ruiz-Jimeno, L. Scodellaro, N. Trevisani, I. Vila,

R. Vilar Cortabitarte

\section{CERN, European Organization for Nuclear Research, Geneva, Switzerland}

D. Abbaneo, E. Auffray, G. Auzinger, P. Baillon, A. H. Ball, D. Barney, P. Bloch, A. Bocci, C. Botta, T. Camporesi,

R. Castello, M. Cepeda, G. Cerminara, Y. Chen, D. d'Enterria, A. Dabrowski, V. Daponte, A. David, M. De Gruttola,

A. De Roeck, E. Di Marco ${ }^{44}$, M. Dobson, B. Dorney, T. du Pree, D. Duggan, M. Dünser, N. Dupont, A. Elliott-Peisert,

P. Everaerts, S. Fartoukh, G. Franzoni, J. Fulcher, W. Funk, D. Gigi, K. Gill, M. Girone, F. Glege, D. Gulhan,

S. Gundacker, M. Guthoff, P. Harris, J. Hegeman, V. Innocente, P. Janot, J. Kieseler, H. Kirschenmann, V. Knünz,

A. Kornmayer ${ }^{16}$, M. J. Kortelainen, K. Kousouris, M. Krammer ${ }^{1}$, C. Lange, P. Lecoq, C. Lourenço, M. T. Lucchini,

L. Malgeri, M. Mannelli, A. Martelli, F. Meijers, J. A. Merlin, S. Mersi, E. Meschi, P. Milenovic ${ }^{45}$, F. Moortgat,

S. Morovic, M. Mulders, H. Neugebauer, S. Orfanelli, L. Orsini, L. Pape, E. Perez, M. Peruzzi, A. Petrilli, G. Petrucciani,

A. Pfeiffer, M. Pierini, A. Racz, T. Reis, G. Rolandi ${ }^{46}$, M. Rovere, H. Sakulin, J. B. Sauvan, C. Schäfer, C. Schwick,

M. Seidel, A. Sharma, P. Silva, P. Sphicas ${ }^{47}$, J. Steggemann, M. Stoye, Y. Takahashi, M. Tosi, D. Treille, A. Triossi,

A. Tsirou, V. Veckalns ${ }^{48}$, G. I. Veres ${ }^{21}$, M. Verweij, N. Wardle, H. K. Wöhri, A. Zagozdzinska ${ }^{36}$, W. D. Zeuner

Paul Scherrer Institut, Villigen, Switzerland

W. Bertl, K. Deiters, W. Erdmann, R. Horisberger, Q. Ingram, H. C. Kaestli, D. Kotlinski, U. Langenegger, T. Rohe, S. A. Wiederkehr

ETH Zurich-Institute for Particle Physics, and Astrophysics (IPA), Zurich, Switzerland

F. Bachmair, L. Bäni, L. Bianchini, B. Casal, G. Dissertori, M. Dittmar, M. Donegà, C. Grab, C. Heidegger, D. Hits, J. Hoss, G. Kasieczka, W. Lustermann, B. Mangano, M. Marionneau, P. Martinez Ruiz del Arbol, M. Masciovecchio, M. T. Meinhard, D. Meister, F. Micheli, P. Musella, F. Nessi-Tedaldi, F. Pandolfi, J. Pata, F. Pauss, G. Perrin, L. Perrozzi, M. Quittnat, M. Rossini, M. Schönenberger, A. Starodumov ${ }^{49}$, V. R. Tavolaro, K. Theofilatos, R. Wallny

Universität Zürich, Zurich, Switzerland

T. K. Aarrestad, C. Amsler ${ }^{50}$, L. Caminada, M. F. Canelli, A. De Cosa, C. Galloni, A. Hinzmann, T. Hreus, B. Kilminster, J. Ngadiuba, D. Pinna, G. Rauco, P. Robmann, D. Salerno, C. Seitz, Y. Yang, A. Zucchetta

National Central University, Chung-Li, Taiwan

V. Candelise, T. H. Doan, Sh. Jain, R. Khurana, M. Konyushikhin, C. M. Kuo, W. Lin, A. Pozdnyakov, S. S. Yu

National Taiwan University (NTU), Taipei, Taiwan

Arun Kumar, P. Chang, Y. H. Chang, Y. Chao, K. F. Chen, P. H. Chen, F. Fiori, W.-S. Hou, Y. Hsiung, Y. F. Liu, R.-S. Lu, M. Miñano Moya, E. Paganis, A. Psallidas, J. f. Tsai

Chulalongkorn University, Faculty of Science, Department of Physics, Bangkok, Thailand

B. Asavapibhop, G. Singh, N. Srimanobhas, N. Suwonjandee

Çukurova University Physics Department, Science and Art Faculty, Adana, Turkey

A. Adiguzel, S. Cerci ${ }^{51}$, S. Damarseckin, Z. S. Demiroglu, C. Dozen, I. Dumanoglu, S. Girgis, G. Gokbulut, Y. Guler, I. $\mathrm{Hos}^{52}$, E. E. Kangal ${ }^{53}$, O. Kara, A. Kayis Topaksu, U. Kiminsu, M. Oglakci, G. Onengut ${ }^{54}$, K. Ozdemir ${ }^{55}$, D. Sunar Cerci $^{51}$, H. Topakli ${ }^{56}$, S. Turkcapar, I. S. Zorbakir, C. Zorbilmez

Middle East Technical University, Physics Department, Ankara, Turkey

B. Bilin, S. Bilmis, B. Isildak ${ }^{57}$, G. Karapinar ${ }^{58}$, M. Yalvac, M. Zeyrek

Bogazici University, Istanbul, Turkey

E. Gülmez, M. Kaya ${ }^{59}$, O. Kaya ${ }^{60}$, E. A. Yetkin ${ }^{61}$, T. Yetkin ${ }^{62}$

Istanbul Technical University, Istanbul, Turkey

A. Cakir, K. Cankocak, S. Sen ${ }^{63}$ 
Institute for Scintillation Materials of National Academy of Science of Ukraine, Kharkov, Ukraine B. Grynyov

National Scientific Center, Kharkov Institute of Physics and Technology, Kharkov, Ukraine

L. Levchuk, P. Sorokin

University of Bristol, Bristol, UK

R. Aggleton, F. Ball, L. Beck, J. J. Brooke, D. Burns, E. Clement, D. Cussans, H. Flacher, J. Goldstein, M. Grimes, G. P. Heath, H. F. Heath, J. Jacob, L. Kreczko, C. Lucas, D. M. Newbold ${ }^{64}$, S. Paramesvaran, A. Poll, T. Sakuma, S. Seif El Nasr-storey, D. Smith, V. J. Smith

\section{Rutherford Appleton Laboratory, Didcot, UK}

K. W. Bell, A. Belyaev ${ }^{65}$, C. Brew, R. M. Brown, L. Calligaris, D. Cieri, D. J. A. Cockerill, J. A. Coughlan, K. Harder, S. Harper, E. Olaiya, D. Petyt, C. H. Shepherd-Themistocleous, A. Thea, I. R. Tomalin, T. Williams

Imperial College, London, UK

M. Baber, R. Bainbridge, O. Buchmuller, A. Bundock, D. Burton, S. Casasso, M. Citron, D. Colling, L. Corpe, P. Dauncey, G. Davies, A. De Wit, M. Della Negra, R. Di Maria, P. Dunne, A. Elwood, D. Futyan, Y. Haddad, G. Hall, G. Iles, T. James, R. Lane, C. Laner, R. Lucas ${ }^{64}$, L. Lyons, A.-M. Magnan, S. Malik, L. Mastrolorenzo, J. Nash, A. Nikitenko ${ }^{49}$, J. Pela, B. Penning, M. Pesaresi, D. M. Raymond, A. Richards, A. Rose, E. Scott, C. Seez, S. Summers, A. Tapper, K. Uchida, M. Vazquez Acosta ${ }^{66}$, T. Virdee ${ }^{16}$, J. Wright, S. C. Zenz

\section{Brunel University, Uxbridge, UK}

J. E. Cole, P. R. Hobson, A. Khan, P. Kyberd, I. D. Reid, P. Symonds, L. Teodorescu, M. Turner

Baylor University, Waco, USA

A. Borzou, K. Call, J. Dittmann, K. Hatakeyama, H. Liu, N. Pastika

Catholic University of America, Washington, DC, USA

R. Bartek, A. Dominguez

The University of Alabama, Tuscaloosa, USA

A. Buccilli, S. I. Cooper, C. Henderson, P. Rumerio, C. West

Boston University, Boston, USA

D. Arcaro, A. Avetisyan, T. Bose, D. Gastler, D. Rankin, C. Richardson, J. Rohlf, L. Sulak, D. Zou

\section{Brown University, Providence, USA}

G. Benelli, D. Cutts, A. Garabedian, J. Hakala, U. Heintz, J. M. Hogan, O. Jesus, K. H. M. Kwok, E. Laird, G. Landsberg, Z. Mao, M. Narain, S. Piperov, S. Sagir, E. Spencer, R. Syarif

\section{University of California, Davis, Davis, USA}

R. Breedon, D. Burns, M. Calderon De La Barca Sanchez, S. Chauhan, M. Chertok, J. Conway, R. Conway, P. T. Cox, R. Erbacher, C. Flores, G. Funk, M. Gardner, W. Ko, R. Lander, C. Mclean, M. Mulhearn, D. Pellett, J. Pilot, S. Shalhout, M. Shi, J. Smith, M. Squires, D. Stolp, K. Tos, M. Tripathi

\section{University of California, Los Angeles, USA}

M. Bachtis, C. Bravo, R. Cousins, A. Dasgupta, A. Florent, J. Hauser, M. Ignatenko, N. Mccoll, D. Saltzberg,

C. Schnaible, V. Valuev, M. Weber

University of California, Riverside, Riverside, USA

E. Bouvier, K. Burt, R. Clare, J. Ellison, J. W. Gary, S. M. A. Ghiasi Shirazi, G. Hanson, J. Heilman, P. Jandir, E. Kennedy, F. Lacroix, O. R. Long, M. Olmedo Negrete, M. I. Paneva, A. Shrinivas, W. Si, H. Wei, S. Wimpenny, B. R. Yates

\section{University of California, San Diego, La Jolla, USA}

J. G. Branson, G. B. Cerati, S. Cittolin, M. Derdzinski, R. Gerosa, A. Holzner, D. Klein, V. Krutelyov, J. Letts, I. Macneill, D. Olivito, S. Padhi, M. Pieri, M. Sani, V. Sharma, S. Simon, M. Tadel, A. Vartak, S. Wasserbaech ${ }^{67}$, C. Welke, J. Wood, F. Würthwein, A. Yagil, G. Zevi Della Porta 


\section{University of California, Santa Barbara-Department of Physics, Santa Barbara, USA}

N. Amin, R. Bhandari, J. Bradmiller-Feld, C. Campagnari, A. Dishaw, V. Dutta, M. Franco Sevilla, C. George, F. Golf,

L. Gouskos, J. Gran, R. Heller, J. Incandela, S. D. Mullin, A. Ovcharova, H. Qu, J. Richman, D. Stuart, I. Suarez, J. Yoo

\section{California Institute of Technology, Pasadena, USA}

D. Anderson, J. Bendavid, A. Bornheim, J. Bunn, J. Duarte, J. M. Lawhorn, A. Mott, H. B. Newman, C. Pena,

M. Spiropulu, J. R. Vlimant, S. Xie, R. Y. Zhu

\section{Carnegie Mellon University, Pittsburgh, USA}

M. B. Andrews, T. Ferguson, M. Paulini, J. Russ, M. Sun, H. Vogel, I. Vorobiev, M. Weinberg

\section{University of Colorado Boulder, Boulder, USA}

J. P. Cumalat, W. T. Ford, F. Jensen, A. Johnson, M. Krohn, S. Leontsinis, T. Mulholland, K. Stenson, S. R. Wagner

\section{Cornell University, Ithaca, USA}

J. Alexander, J. Chaves, J. Chu, S. Dittmer, K. Mcdermott, N. Mirman, G. Nicolas Kaufman, J. R. Patterson,

A. Rinkevicius, A. Ryd, L. Skinnari, L. Soffi, S. M. Tan, Z. Tao, J. Thom, J. Tucker, P. Wittich, M. Zientek

\section{Fairfield University, Fairfield, USA}

D. Winn

\section{Fermi National Accelerator Laboratory, Batavia, USA}

S. Abdullin, M. Albrow, G. Apollinari, A. Apresyan, S. Banerjee, L. A. T. Bauerdick, A. Beretvas, J. Berryhill, P. C. Bhat, G. Bolla, K. Burkett, J. N. Butler, H. W. K. Cheung, F. Chlebana, S. Cihangir ${ }^{\dagger}$, M. Cremonesi, V. D. Elvira, I. Fisk,

J. Freeman, E. Gottschalk, L. Gray, D. Green, S. Grünendahl, O. Gutsche, D. Hare, R. M. Harris, S. Hasegawa,

J. Hirschauer, Z. Hu, B. Jayatilaka, S. Jindariani, M. Johnson, U. Joshi, B. Klima, B. Kreis, S. Lammel, J. Linacre,

D. Lincoln, R. Lipton, M. Liu, T. Liu, R. Lopes De Sá, J. Lykken, K. Maeshima, N. Magini, J. M. Marraffino,

S. Maruyama, D. Mason, P. McBride, P. Merkel, S. Mrenna, S. Nahn, V. O’Dell, K. Pedro, O. Prokofyev, G. Rakness,

L. Ristori, E. Sexton-Kennedy, A. Soha, W. J. Spalding, L. Spiegel, S. Stoynev, J. Strait, N. Strobbe, L. Taylor, S. Tkaczyk,

N. V. Tran, L. Uplegger, E. W. Vaandering, C. Vernieri, M. Verzocchi, R. Vidal, M. Wang, H. A. Weber, A. Whitbeck, Y. Wu

\section{University of Florida, Gainesville, USA}

D. Acosta, P. Avery, P. Bortignon, D. Bourilkov, A. Brinkerhoff, A. Carnes, M. Carver, D. Curry, S. Das, R. D. Field,

I. K. Furic, J. Konigsberg, A. Korytov, J. F. Low, P. Ma, K. Matchev, H. Mei, G. Mitselmakher, D. Rank, L. Shchutska,

D. Sperka, L. Thomas, J. Wang, S. Wang, J. Yelton

\section{Florida International University, Miami, USA}

S. Linn, P. Markowitz, G. Martinez, J. L. Rodriguez

Florida State University, Tallahassee, USA

A. Ackert, T. Adams, A. Askew, S. Bein, S. Hagopian, V. Hagopian, K. F. Johnson, T. Kolberg, T. Perry, H. Prosper, A. Santra, R. Yohay

\section{Florida Institute of Technology, Melbourne, USA}

M. M. Baarmand, V. Bhopatkar, S. Colafranceschi, M. Hohlmann, D. Noonan, T. Roy, F. Yumiceva

\section{University of Illinois at Chicago (UIC), Chicago, USA}

M. R. Adams, L. Apanasevich, D. Berry, R. R. Betts, I. Bucinskaite, R. Cavanaugh, X. Chen, O. Evdokimov, L. Gauthier, C. E. Gerber, D. J. Hofman, K. Jung, I. D. Sandoval Gonzalez, N. Varelas, H. Wang, Z. Wu, M. Zakaria, J. Zhang

The University of Iowa, Iowa City, USA

B. Bilki ${ }^{68}$, W. Clarida, K. Dilsiz, S. Durgut, R. P. Gandrajula, M. Haytmyradov, V. Khristenko, J.-P. Merlo, H. Mermerkaya ${ }^{69}$, A. Mestvirishvili, A. Moeller, J. Nachtman, H. Ogul, Y. Onel, F. Ozok ${ }^{70}$, A. Penzo, C. Snyder, E. Tiras, J. Wetzel, K. Yi

\section{Johns Hopkins University, Baltimore, USA}

B. Blumenfeld, A. Cocoros, N. Eminizer, D. Fehling, L. Feng, A. V. Gritsan, P. Maksimovic, J. Roskes, U. Sarica, M. Swartz, M. Xiao, C. You 
The University of Kansas, Lawrence, USA

A. Al-bataineh, P. Baringer, A. Bean, S. Boren, J. Bowen, J. Castle, L. Forthomme, R. P. Kenny III, S. Khalil,

A. Kropivnitskaya, D. Majumder, W. Mcbrayer, M. Murray, S. Sanders, R. Stringer, J. D. Tapia Takaki, Q. Wang

Kansas State University, Manhattan, USA

A. Ivanov, K. Kaadze, Y. Maravin, A. Mohammadi, L. K. Saini, N. Skhirtladze, S. Toda

Lawrence Livermore National Laboratory, Livermore, USA

F. Rebassoo, D. Wright

University of Maryland, College Park, USA

C. Anelli, A. Baden, O. Baron, A. Belloni, B. Calvert, S. C. Eno, C. Ferraioli, J. A. Gomez, N. J. Hadley, S. Jabeen,

G. Y. Jeng, R. G. Kellogg, J. Kunkle, A. C. Mignerey, F. Ricci-Tam, Y. H. Shin, A. Skuja, M. B. Tonjes, S. C. Tonwar

Massachusetts Institute of Technology, Cambridge, USA

D. Abercrombie, B. Allen, A. Apyan, V. Azzolini, R. Barbieri, A. Baty, R. Bi, K. Bierwagen, S. Brandt, W. Busza,

I. A. Cali, M. D'Alfonso, Z. Demiragli, G. Gomez Ceballos, M. Goncharov, D. Hsu, Y. Iiyama, G. M. Innocenti, M. Klute,

D. Kovalskyi, K. Krajczar, Y. S. Lai, Y.-J. Lee, A. Levin, P. D. Luckey, B. Maier, A. C. Marini, C. Mcginn, C. Mironov,

S. Narayanan, X. Niu, C. Paus, C. Roland, G. Roland, J. Salfeld-Nebgen, G. S. F. Stephans, K. Tatar, D. Velicanu, J. Wang,

T. W. Wang, B. Wyslouch

University of Minnesota, Minneapolis, USA

A. C. Benvenuti, R. M. Chatterjee, A. Evans, P. Hansen, S. Kalafut, S. C. Kao, Y. Kubota, Z. Lesko, J. Mans,

S. Nourbakhsh, N. Ruckstuhl, R. Rusack, N. Tambe, J. Turkewitz

University of Mississippi, Oxford, USA

J. G. Acosta, S. Oliveros

University of Nebraska-Lincoln, Lincoln, USA

E. Avdeeva, K. Bloom, D. R. Claes, C. Fangmeier, R. Gonzalez Suarez, R. Kamalieddin, I. Kravchenko,

A. Malta Rodrigues, J. Monroy, J. E. Siado, G. R. Snow, B. Stieger

State University of New York at Buffalo, Buffalo, USA

M. Alyari, J. Dolen, A. Godshalk, C. Harrington, I. Iashvili, J. Kaisen, D. Nguyen, A. Parker, S. Rappoccio, B. Roozbahani

Northeastern University, Boston, USA

G. Alverson, E. Barberis, A. Hortiangtham, A. Massironi, D. M. Morse, D. Nash, T. Orimoto, R. Teixeira De Lima,

D. Trocino, R.-J. Wang, D. Wood

Northwestern University, Evanston, USA

S. Bhattacharya, O. Charaf, K. A. Hahn, A. Kumar, N. Mucia, N. Odell, B. Pollack, M. H. Schmitt, K. Sung, M. Trovato, M. Velasco

University of Notre Dame, Notre Dame, USA

N. Dev, M. Hildreth, K. Hurtado Anampa, C. Jessop, D. J. Karmgard, N. Kellams, K. Lannon, N. Marinelli, F. Meng, C. Mueller, Y. Musienko ${ }^{37}$, M. Planer, A. Reinsvold, R. Ruchti, N. Rupprecht, G. Smith, S. Taroni, M. Wayne, M. Wolf,

A. Woodard

The Ohio State University, Columbus, USA

J. Alimena, L. Antonelli, B. Bylsma, L. S. Durkin, S. Flowers, B. Francis, A. Hart, C. Hill, W. Ji, B. Liu, W. Luo,

D. Puigh, B. L. Winer, H. W. Wulsin

Princeton University, Princeton, USA

S. Cooperstein, O. Driga, P. Elmer, J. Hardenbrook, P. Hebda, D. Lange, J. Luo, D. Marlow, T. Medvedeva, K. Mei,

I. Ojalvo, J. Olsen, C. Palmer, P. Piroué, D. Stickland, A. Svyatkovskiy, C. Tully

University of Puerto Rico, Mayaguez, USA

S. Malik 


\section{Purdue University, West Lafayette, USA}

A. Barker, V. E. Barnes, S. Folgueras, L. Gutay, M. K. Jha, M. Jones, A. W. Jung, A. Khatiwada, D. H. Miller,

N. Neumeister, J. F. Schulte, X. Shi, J. Sun, F. Wang, W. Xie

Purdue University Northwest, Hammond, USA

N. Parashar, J. Stupak

Rice University, Houston, USA

A. Adair, B. Akgun, Z. Chen, K. M. Ecklund, F. J. M. Geurts, M. Guilbaud, W. Li, B. Michlin, M. Northup, B. P. Padley, J. Roberts, J. Rorie, Z. Tu, J. Zabel

University of Rochester, Rochester, USA

B. Betchart, A. Bodek, P. de Barbaro, R. Demina, Y. t. Duh, T. Ferbel, M. Galanti, A. Garcia-Bellido, J. Han, O. Hindrichs, A. Khukhunaishvili, K. H. Lo, P. Tan, M. Verzetti

\section{The Rockefeller University, New York, USA}

R. Ciesielski

Rutgers, The State University of New Jersey, Piscataway, USA

A. Agapitos, J. P. Chou, Y. Gershtein, T. A. Gómez Espinosa, E. Halkiadakis, M. Heindl, E. Hughes, S. Kaplan,

R. Kunnawalkam Elayavalli, S. Kyriacou, A. Lath, K. Nash, M. Osherson, H. Saka, S. Salur, S. Schnetzer, D. Sheffield,

S. Somalwar, R. Stone, S. Thomas, P. Thomassen, M. Walker

University of Tennessee, Knoxville, USA

A. G. Delannoy, M. Foerster, J. Heideman, G. Riley, K. Rose, S. Spanier, K. Thapa

Texas A\&M University, College Station, USA

O. Bouhali ${ }^{71}$, A. Celik, M. Dalchenko, M. De Mattia, A. Delgado, S. Dildick, R. Eusebi, J. Gilmore, T. Huang, E. Juska,

T. Kamon ${ }^{72}$, R. Mueller, Y. Pakhotin, R. Patel, A. Perloff, L. Perniè, D. Rathjens, A. Safonov, A. Tatarinov, K. A. Ulmer

Texas Tech University, Lubbock, USA

N. Akchurin, J. Damgov, F. De Guio, C. Dragoiu, P. R. Dudero, J. Faulkner, E. Gurpinar, S. Kunori, K. Lamichhane,

S. W. Lee, T. Libeiro, T. Peltola, S. Undleeb, I. Volobouev, Z. Wang

Vanderbilt University, Nashville, USA

S. Greene, A. Gurrola, R. Janjam, W. Johns, C. Maguire, A. Melo, H. Ni, P. Sheldon, S. Tuo, J. Velkovska, Q. Xu

University of Virginia, Charlottesville, USA

M. W. Arenton, P. Barria, B. Cox, J. Goodell, R. Hirosky, A. Ledovskoy, H. Li, C. Neu, T. Sinthuprasith, X. Sun, Y. Wang, E. Wolfe, F. Xia

Wayne State University, Detroit, USA

C. Clarke, R. Harr, P. E. Karchin, J. Sturdy, S. Zaleski

University of Wisconsin-Madison, Madison, WI, USA

D. A. Belknap, J. Buchanan, C. Caillol, S. Dasu, L. Dodd, S. Duric, B. Gomber, M. Grothe, M. Herndon, A. Hervé, P. Klabbers, A. Lanaro, A. Levine, K. Long, R. Loveless, G. A. Pierro, G. Polese, T. Ruggles, A. Savin, N. Smith, W. H. Smith, D. Taylor, N. Woods

\section{$\dagger$ Deceased}

1: Also at Vienna University of Technology, Vienna, Austria

2: Also at State Key Laboratory of Nuclear Physics and Technology, Peking University, Beijing, China

3: Also at Institut Pluridisciplinaire Hubert Curien (IPHC), Université de Strasbourg, CNRS/IN2P3, Strasbourg, France

4: Also at Universidade Estadual de Campinas, Campinas, Brazil

5: Also at Universidade Federal de Pelotas, Pelotas, Brazil

6: Also at Université Libre de Bruxelles, Bruxelles, Belgium

7: Also at Deutsches Elektronen-Synchrotron, Hamburg, Germany

8: Also at Universidad de Antioquia, Medellin, Colombia

9: Also at Joint Institute for Nuclear Research, Dubna, Russia 
10: Now at British University in Egypt, Cairo, Egypt

11: Now at Cairo University, Cairo, Egypt

12: Also at Zewail City of Science and Technology, Zewail, Egypt

13: Also at Université de Haute Alsace, Mulhouse, France

14: Also at Skobeltsyn Institute of Nuclear Physics, Lomonosov Moscow State University, Moscow, Russia

15: Also at Tbilisi State University, Tbilisi, Georgia

16: Also at CERN, European Organization for Nuclear Research, Geneva, Switzerland

17: Also at RWTH Aachen University, III. Physikalisches Institut A, Aachen, Germany

18: Also at University of Hamburg, Hamburg, Germany

19: Also at Brandenburg University of Technology, Cottbus, Germany

20: Also at Institute of Nuclear Research ATOMKI, Debrecen, Hungary

21: Also at MTA-ELTE Lendület CMS Particle and Nuclear Physics Group, Eötvös Loránd University, Budapest, Hungary

22: Also at Institute of Physics, University of Debrecen, Debrecen, Hungary

23: Also at Indian Institute of Technology Bhubaneswar, Bhubaneswar, India

24: Also at University of Visva-Bharati, Santiniketan, India

25: Also at Indian Institute of Science Education and Research, Bhopal, India

26: Also at Institute of Physics, Bhubaneswar, India

27: Also at University of Ruhuna, Matara, Sri Lanka

28: Also at Isfahan University of Technology, Isfahan, Iran

29: Also at Yazd University, Yazd, Iran

30: Also at Plasma Physics Research Center, Science and Research Branch, Islamic Azad University, Tehran, Iran

31: Also at Università degli Studi di Siena, Siena, Italy

32: Also at Purdue University, West Lafayette, USA

33: Also at International Islamic University of Malaysia, Kuala Lumpur, Malaysia

34: Also at Malaysian Nuclear Agency, MOSTI, Kajang, Malaysia

35: Also at Consejo Nacional de Ciencia y Tecnología, Mexico city, Mexico

36: Also at Warsaw University of Technology, Institute of Electronic Systems, Warsaw, Poland

37: Also at Institute for Nuclear Research, Moscow, Russia

38: Now at National Research Nuclear University 'Moscow Engineering Physics Institute' (MEPhI), Moscow, Russia

39: Also at St. Petersburg State Polytechnical University, St. Petersburg, Russia

40: Also at University of Florida, Gainesville, USA

41: Also at P.N. Lebedev Physical Institute, Moscow, Russia

42: Also at Budker Institute of Nuclear Physics, Novosibirsk, Russia

43: Also at Faculty of Physics, University of Belgrade, Belgrade, Serbia

44: Also at INFN Sezione di Roma; Sapienza Università di Roma, Rome, Italy

45: Also at University of Belgrade, Faculty of Physics and Vinca Institute of Nuclear Sciences, Belgrade, Serbia

46: Also at Scuola Normale e Sezione dell'INFN, Pisa, Italy

47: Also at National and Kapodistrian University of Athens, Athens, Greece

48: Also at Riga Technical University, Riga, Latvia

49: Also at Institute for Theoretical and Experimental Physics, Moscow, Russia

50: Also at Albert Einstein Center for Fundamental Physics, Bern, Switzerland

51: Also at Adiyaman University, Adiyaman, Turkey

52: Also at Istanbul Aydin University, Istanbul, Turkey

53: Also at Mersin University, Mersin, Turkey

54: Also at Cag University, Mersin, Turkey

55: Also at Piri Reis University, Istanbul, Turkey

56: Also at Gaziosmanpasa University, Tokat, Turkey

57: Also at Ozyegin University, Istanbul, Turkey

58: Also at Izmir Institute of Technology, Izmir, Turkey

59: Also at Marmara University, Istanbul, Turkey

60: Also at Kafkas University, Kars, Turkey

61: Also at Istanbul Bilgi University, Istanbul, Turkey

62: Also at Yildiz Technical University, Istanbul, Turkey 
63: Also at Hacettepe University, Ankara, Turkey

64: Also at Rutherford Appleton Laboratory, Didcot, UK

65: Also at School of Physics and Astronomy, University of Southampton, Southampton, UK

66: Also at Instituto de Astrofísica de Canarias, La Laguna, Spain

67: Also at Utah Valley University, Orem, USA

68: Also at Argonne National Laboratory, Argonne, USA

69: Also at Erzincan University, Erzincan, Turkey

70: Also at Mimar Sinan University, Istanbul, Istanbul, Turkey

71: Also at Texas A\&M University at Qatar, Doha, Qatar

72: Also at Kyungpook National University, Taegu, Korea 